\title{
Encouraging general dentists to expand their comprehensive capabilities
}

Align Technology, Inc. has announced the availability of a new page in the Invisalign Go website (https://www.invisalign-go.co.uk/ learning) for general dentists - both existing Invisalign providers, as well as those keen to expand their comprehensive treatment capabilities.

Through this new page, Align provides general dentists with information about how to integrate Invisalign clear aligners into their comprehensive treatment plans, with the added benefits of precision, exceptional outcomes and a seamless digital workflow. Dentists can learn more about clear aligner therapy used as a way to offer minimally invasive care - as part of an ortho-restorative process as a corrected bite promotes the ideal environment for precise, reliable results, with no need for aggressive tooth reduction.

Learning by example is important so there is an Invisalign Go outcome gallery on the site and dentists can also request gold standard examples of cases which will show them how to integrate Invisalign treatments into their clinical practice. Also accessible is a free copy of Align's Best Practice Statement describing 'The Diagnosis and Treatment of Malocclusion', which explains how orthodontic treatment can prevent and intercept oral diseases and improve quality of life. ${ }^{1}$
Because the Invisalign system is only part of the picture, there is a section on the page about mastering efficiencies with a seamless digital workflow, which highlights the use of the iTero digital scanner and some of the new tools Align has created for its integrated digital platform.

Know-how from experts is reassuring so there are also videos on the site from respected dentists who describe how the Invisalign system and digital workflow amplifies the effectiveness of their comprehensive dentistry protocols. These are all general dentists from across the world who have been successfully incorporating Invisalign clear aligners as part of their treatment plans, and have also been invited to share their stories, experiences and case studies on the new platform.

News about Invisalign certification courses, continued education and Align staged events are also detailed on the site and it is possible to sign up to these with a single click.

For more information, visit https://www.invisalign-go.co.uk/ learning.

\section{Reference}

1. Pacheco-Pereira C, Brandelli J, Flores-Mir C. Patient satisfaction and quality of life changes after Invisalign treatment. Am J Orthod Dentofacial Orthop 2018; 153: 834-841

\section{Introducing the next generation of intraoral}

\section{scanner}
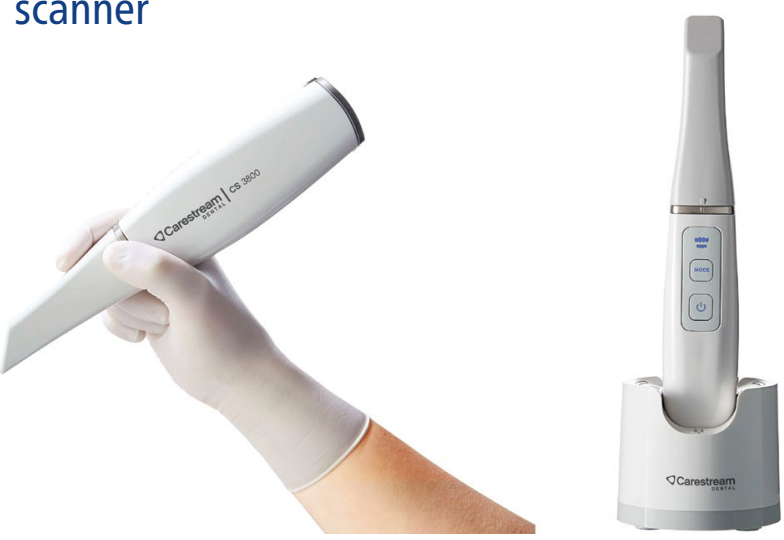

Innovative. Versatile. Easy-to-use.

These are just three ways to describe the new CS 3800 wireless intraoral scanner from Carestream Dental.

A system at the very cutting-edge of diagnostic excellence, the CS 3800 wireless features robust and seamless wireless capabilities with up to one hour of continuous scanning. Designed for superior user comfort and functionality, the system can perform a turbo speed scan of a single arch in just 25 seconds [internal testing completed on a 3D model. Scanning time may vary based on the tooth model and PC].

Plus, the scanner is highly accurate, meaning that professionals can rely on super-quick, crystal clear images every time!

Find out how the CS 3800 wireless can make a difference for you by contacting the team today.

For more information, contact Carestream Dental on 0800169 9692 or visit www.carestreamdental.co.uk.

\section{Book a test drive today!}

The Axiom Multi Level implant system from Anthogyr provides a highly versatile solution designed to optimise predictability and accessibility of treatment. It contains both tissue level and bone level implants with a singular surgery kit for workflow simplicity.

You can now book an Anthogyr Test Drive to see how the system works in your own hands. The programme includes:

- One day use of the Axiom Surgical Kit

- One complementary Anthogyr implant

- Support from a Territory Manager on the day of surgery

- Half price restorative components on selected items.

Available through the Straumann Group, clinicians can have complete confidence in the science behind the Anthogyr implant system and the quality of support provided from a highly knowledgeable and accessible team. Find out more today.

For more information and to book your Anthogyr Test Drive, visit www.anthogyr-uk.co/testdrive.

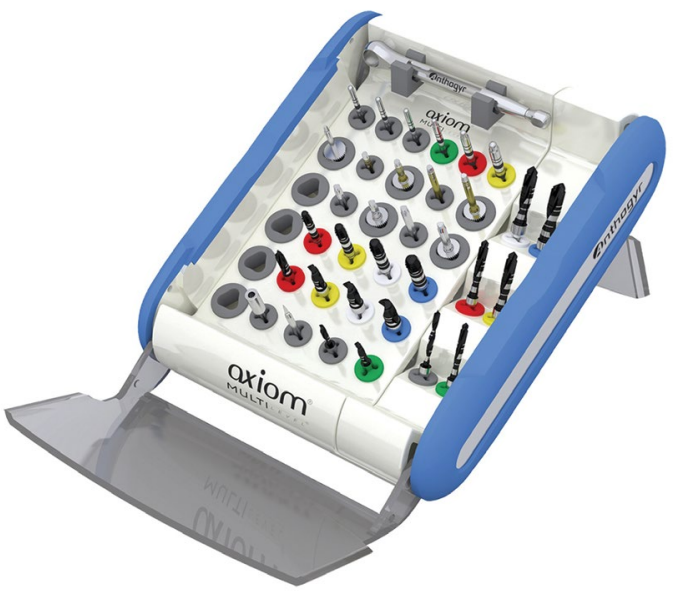

